\title{
Lotsen durch die Rehabilitation
}

\author{
Anett Reimann und Jutta Spormann
}

\begin{abstract}
Menschen mit Unfallverletzungen oder einer chronischen Krankheit finden sich oft nicht im gegliederten Sicherungssystem zurecht. Unabhängige Lotsen können hier als Case Manager zusammen mit allen Beteiligten den im Einzelfall besten Weg zur Wiedereingliederung finden, wie das Beispiel des bundesweit tätigen Unternehmens InReha zeigt.
\end{abstract}

Das soziale Sicherungssystem in der Bundesrepublik Deutschland ist vielfältig und schwer überschaubar. Die Rehabilitation ist auf Grundlage der in den Sozialgesetzen verankerten Bestimmungen umfassend geregelt und bietet per se beste Chancen für den betroffenen Menschen.

Im zergliederten System der Hilfen in Deutschland ist die Realität jedoch oft eine andere: Ein Zusammenwirken der unterschiedlichen Leistungsträger, koordiniertes Handeln und Absprachen geschehen nur selten. Trotz eines auf der gesetzlichen Ebene erfolgten Paradigmenwechsels, etwa mit der Einführung des Gesetzes zur Rehabilitation und Teilhabe behinderter Menschen (SGB IX), hat sich in den letzten Jahren im Hinblick auf die Optimierung der Prozesse nur wenig geändert.

Bereits in den Jahren 1998 bis 2001 wurde in SchleswigHolstein das Vernetzungsprojekt »WiederEingliederungsGemeinschaft (WEG) « zwischen Rehabilitationskliniken, Integrationsfachdiensten und beteiligten Kostenträgern als Modellprojekt im Auftrag des Bundesarbeitsministeriums umgesetzt. Ziel war eine nahtlose berufliche (Wieder-) Eingliederung der Patienten. Kompetenzen der beteiligten Kliniken sollten mit denen der ebenfalls beteiligten Integrationsfachdienste verbunden werden. Ein Steuerungsteam bestehend aus Vertretern der Arbeitsagenturen, Rentenversicherern und Krankenkassen, das fortlaufend Informationen zum Rehabilitationsverlauf erhielt, sollte in Abstimmung mit den Beteiligten zeitnah Entscheidungen treffen, die den Fortgang des Rehabilitationsprozesses ermöglichten.

Gerade wegen seines fast revolutionären Ansatzes war das Projekt ohnmächtig gegen die allgegenwärtigen Partikularinteressen und ungeeigneten Entscheidungsstrukturen bei den Kostenträgern. In der Konsequenz wurde der Versuch, ein überregionales Netzwerk zu errichten, aufgegeben. Stattdessen wurde »zwischen Krankenbett und Arbeitsplatz« ein konsequentes Case Management eingeführt (Wolf Rainer Wendt: Case Management im Sozialund Gesundheitswesen. Lambertus, Freiburg im Breisgau 1997). Diese Maßnahme belegte im Ergebnis: Die Integration chronisch erkrankter Menschen kann auch in-

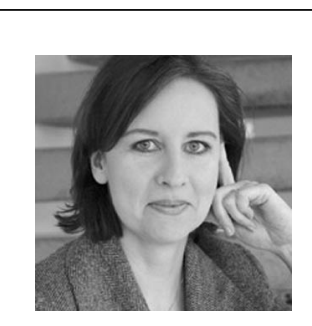

Jutta Spormann, Diplom-Betriebswirtin und Master of International Business Administration, ist bei InReha tätig im Projekt Gesunde Arbeit Schleswig-Holstein. Ihre Schwerpunkte der letzten Jahre lagen in der beruflichen Rehabilitation, im Personalwesen sowie im Bereich Corporate Social Responsibility. E-Mail jutta.spormann@gesundearbeit-sh.de

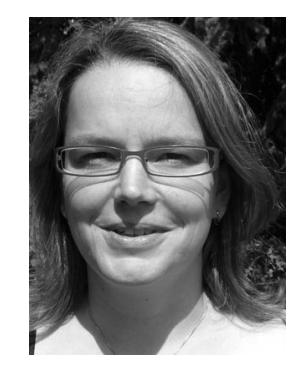

Anett Reimann, Diplom-Heilpädagogin und Certified Disability Management Professional (CDMP), ist bei InReha als freie Mitarbeiterin tätig in der zentralen Koordination, ebenso als Fallmanagerin. Schwerpunkte der Tätigkeit: medizinische und berufliche Rehabilitation mit Fokus im Bereich Schädel-Hirn-Verletzungen.

nerhalb widriger Strukturen erfolgreich verlaufen, wenn konsequent nach Case-Management-Kriterien verfahren wird.

\section{Ein gemeinsames Ziel: Höchstmaß an Teilhabe}

Der beste Beweis ist die Tat - seit 2001 setzt »InReha « als unabhängiger Rehabilitationsdienst konsequent das fort, was im Projekt WEG durch »Querdenken « erfolgreich erprobt wurde. InReha ist ein bundesweit tätiges Unternehmen mit Sitz in Hamburg. In der Hamburger Zentrale steuern Koordinatoren die Fallbegleitung und sind Ansprechpartner, Berater, Supervisoren für die jeweils vor Ort tätigen Fallbegleiter, die direkt mit dem Rehabilitanden arbeiten. Hohe fachliche Eignung, die Fähigkeit zu vernetztem Denken und Handeln sowie umfangreiche Erfahrungen im Bereich der Rehabilitation, ebenso exzellentes Kommunikationsvermögen und Moderationskompetenz sind Voraussetzungen für eine Tätigkeit in diesem komplexen Arbeitsfeld. Das eingesetzte Steuerungsverfahren heißt hier »Integratives Fallmanagement «. Zentrale Punkte dieses Verfahrens sind Interdisziplinarität, Ressourcenorientierung, Individualität und nicht zuletzt Kreativität - das alles mit dem Ziel, mit und für den Rehabilitanden die größtmögliche Teilhabe zu erreichen. Es 
richtet sich an Menschen, deren Lebensweg (-planung) durch ein Unfallereignis oder eine schwere Erkrankung unterbrochen wurde.

Hauptaufgabenfeld von InReha sind dabei Integrationsbegleitungen, die im Idealfall zeitnah nach einem Unfallereignis, also bereits in der Akutphase, beginnen, die Phase der stationären Rehabilitation und anschließenden ambulanten Rehabilitation beinhalten und mit der sozialen und beruflichen Integration enden. Auftraggeber solcher umfassenden Maßnahmen sind in erster Linie Kraftfahrthaftpflichtversicherer; Teilbereiche werden durch Berufsgenossenschaften oder die Rentenversicherung beauftragt.

\section{Wie InReha arbeitet}

Skizziert für den Einzelfall bedeutet das folgenden Ablauf: Der jeweilige Auftraggeber schaltet InReha ein und stellt mit dem Einverständnis des Betroffenen erste vorliegende medizinische Unterlagen zur Verfügung. Als Ziel wird dabei oftmals die Klärung der aktuellen Situation und Unterstützung bei der Steuerung der medizinischen und beruflichen Rehabilitationsleistungen formuliert. Über die InReha-Zentrale wird vor Ort ein geeigneter Mitarbeiter eingeschaltet, der dann ein Erstgespräch mit dem Betroffenen und gegebenenfalls dessen Angehörigen führt, oft unter Einbeziehung des beteiligten Rechtsanwaltes. Ausführliche Informationen zur medizinischen Situation werden bei Vorliegen einer Schweigepflichtentbindung von den beteiligten Behandlern ebenfalls eingeholt. Auf der Basis einer gründlichen Anamnese wird dann mit dem Betroffenen gemeinsam eine Eingliederungszielplanung erarbeitet, die das Ziel und gleichermaßen die Richtung des Rehabilitationsweges bestimmt. Diese Planung ist kein statisches Instrument, sondern wird im Sinne der Prozesshaftigkeit stetig auf Aktualität und erforderliche Anpassungen hin überprüft.

\section{InReha}

steht für individuelle Rehabilitation. Seit 2001 begleitet das Unternehmen bundesweit Menschen mit schweren Unfallverletzungen bei der medizinischen, beruflichen, schulischen und sozialen Rehabilitation. Die 250 regionalen Integrationsbegleiter sind überall in Deutschland tätig. Sie verfügen über langjährige Erfahrungen in der Gestaltung der Rehabilitation von Menschen auch mit besonders schweren Beeinträchtigungen wie Schädelhirnverletzungen und psychischen Traumatisierungen. InReha unterstützt Sie und Ihre Angehörigen durch individuelle Beratung und koordiniert geeignete Maßnahmen.

InReha GmbH, Partner für neue Ziele, Havighorster Weg 8a, 21031 Hamburg, Telefon 040 720040-80, Fax 040 720040-88, E-Mail info@inreha.net, Internet http://www.inreha.net
Im weiteren Verlauf ist es Aufgabe des vor Ort tätigen Fallmanagers, die Kommunikation zwischen den unterschiedlichen Beteiligten (Betroffenem, Angehörigen, Ärzte und Therapeuten, Kostenträger, Arbeitgeber etc.) zu ermöglichen und somit sicherzustellen, dass die vereinbarten Reha-Ziele umgesetzt werden. Gemeinsam werden erforderliche Therapien und Behandlungen abgestimmt, Kostenübernahmen geklärt und Terminabstimmungen getroffen. Zu spezifischen Fragestelllungen, beispielsweise weiterführender Diagnostik der kognitiven und körperlichen Leistungsfähigkeit, Abklärung von Pflegebedarf, Umbaumaßnahmen, Mobilitätshilfen u. v. a. m., schaltet InReha Experten, wie Neuropsychologen, Architekten mit Schwerpunkt barrierefreies Bauen, Spezialkliniken, Fachdienste etc. ein, mit deren Hilfe individuelle Ergebnisse, Informationen und Empfehlungen erarbeitet werden, die im weiteren Verlauf zur Umsetzung kommen.

Die Zielstellung des Erreichens größtmöglicher Teilhabe für den Rehabilitanden ist von Fall zu Fall verschieden. Neben beruflicher (Wieder-) Eingliederung kann auch das Erreichen bestmöglicher Selbständigkeit im Alltag Ziel der Bemühungen sein. Je nach Einzelfall geht es also u. a. darum, Arbeits- oder Ausbildungsoptionen zu erarbeiten, vorhandene Arbeitsplätze ressourcenorientiert anzupassen, Nischenarbeitsplätze zu schaffen, barrierefreie Baumaßnahmen umzusetzen oder technische Hilfsmittel zu installieren.

\section{"Menschen und Unternehmen}

\section{erwarten von Gesundheitsdienstleitern unbürokratische und zügige Hilfe- alles wie aus einer Hand «}

Stets gilt es, den Behandlungs- und Rehabilitationspfad so individuell wie möglich zu gestalten und dabei ergänzende Fachkompetenz adäquat zu integrieren. Im Bedarfsfall werden also externe Spezialisten eingebunden, um das Reha-Team passgenau zu ergänzen. InReha agiert dabei als Kompetenznetzwerk, in das Mitarbeiter und externe Fachkräfte aus den Bereichen Medizin, Beruf, Schule, Pflege, Mobilität und Wohnen ihre fachlichen Kenntnisse einbringen. Der Fallmanager vor Ort fungiert als Lotse, der den Rehabilitanden dabei unterstützt, seinen Weg durch das gelegentlich undurchsichtige, verzweigte System der Hilfen zu finden und sicher zu gehen.

\section{Unternehmer-Lotse für gesunde Arbeit}

Dass diese Lotsenfunktion nicht nur Rehabilitanden mit Unfallverletzungen oder chronischen Erkrankungen weiter hilft, beweist ein Modellprojekt, an dem InReha beteiligt ist. Unternehmer oder Personalverantwortliche in kleinen oder mittelgroßen Betrieben stehen bei Fragen zur Gesundheit am Arbeitsplatz immer wieder vor Problemen. In den Unternehmen selbst sind dazu oft weder entsprechendes Fachwissen noch geeignete Strukturen vorhanden. Ein unbürokratischer, einfacher und verlässlicher 
Zugang zu den im Sozialversicherungssystem gegebenen Möglichkeiten der Unterstützung besteht nach wie vor nicht. Angesicht der mit dem demografischen Wandel einhergehenden Veränderungen geraten gerade die kleinen und mittleren Unternehmen in Gefahr, abgehängt zu werden.

Das bundesweite Modellprojekt »Gesunde Arbeit « des Bundesministeriums für Arbeit und Soziales hat die Verbesserung der Beschäftigungsfähigkeit in kleinen und mittleren Unternehmen zum Ziel (Internet

http://www.gesunde-arbeit.net). InReha konnte dabei als Projektträger in Schleswig-Holstein nachweisen, dass Unternehmer in gleicher Weise wie Unfallverletzte von unabhängigen Lotsen profitieren. Gleichermaßen nutzt die vom Lotsen geleistete Koordinierung den Sozialversicherungsträgern ebenso wie den Anbietern von Gesundheitsdienstleistungen, deren Angebote von Unternehmen besser wahrgenommen und zielgenauer eingesetzt werden. Mit einer Lotsenstelle und einem systematischen Netzwerkaufbau zwischen den beteiligten Trägern kann unter der Nutzung von Synergien trägerübergreifend ein geeignetes Unterstützungsangebot für kleine und mittlere Unternehmen vorgehalten werden, das den Erwartungen der Betriebe entspricht: unbürokratisch, zügig, alles wie aus einer Hand.

Ein trägerübergreifend koordiniertes betriebliches Gesundheitsmanagement in kleinen und mittleren Unternehmen ist für alle beteiligten Träger Neuland und somit Lernfeld. Durch die Beteiligung der Träger der Unfallversicherung (Arbeits- und Gesundheitsschutz, betriebliches Eingliederungsmanagement), der Krankenkassen (betriebliche Gesundheitsförderung nach § 20 SGB V) und der Rentenversicherungsträger (z. B. bei Qualifizierungsangeboten für ältere Arbeitnehmer) gelingt es, kleine und mittlere Unternehmen bei überschaubarem Mitteleinsatz auf eine Zukunft als gesunder Betrieb vorzubereiten.

Trotz mancher Schwierigkeiten und nicht ausgebliebener Rückschläge hat das Projekt »Gesunde Arbeit « in Schleswig-Holstein in den vergangenen zwei Jahren durch eine konsequente Vernetzung auf Basis eines Case Managements auf der Systemebene (vgl. H. Mennemann, Case Management auf der Systemebene - Aufbau von Netzwerken, in: Case Management 1/2006) eine landesweite Verbundstruktur mit allen wichtigen Sozialversicherungsträgern aufgebaut, die nun weiter gefestigt, verankert und ausgebaut werden soll. Es handelt sich um ein ambitio- niertes Projekt, das eine im Sinne des Gemeinwesens verbesserte Zusammenarbeit der Beteiligten ermöglichen und so einen sozialwirtschaftlichen Nutzen stiften soll. Die nachhaltige Umsetzung wird wesentlich davon abhängen, inwieweit alle Beteiligten bereit sind, bestehende Partialinteressen zurückzustellen.

Nach nunmehr acht Jahren konsequenter Arbeit als unabhängiger Rehabilitationsdienst ist InReha überzeugt: Unabhängige Lotsen und aktive Netzwerke sind im Rehabilitationssystem unerlässlich, um Kräfte zu bündeln, quer zu denken und klar zu handeln, um für alle Beteiligten die möglichst adäquate Versorgung zu erreichen und darüber eine Rehabilitation zu ermöglichen, die im Sinne aller Beteiligten bestmögliche Erfolge sichert.

Diese Erkenntnis gilt uneingeschränkt für alle Menschen mit unfall- oder erkrankungsbedingten Einschränkungen der individuellen Leistungsfähigkeit gleichermaßen wie für Unternehmer mit älter und kränker werdenden Arbeitnehmern - unabhängig von der jeweiligen Kostenträgerschaft. In diese Lotsenleistungen investierte finanzielle Mittel sind Ausgaben, die - gut abgestimmt und sinnvoll eingesetzt - dabei helfen, mittelund langfristig Kosten deutlich zu senken.

»Die Integration und selbstbestimmte Teilhabe behinderter Menschen am sozialen, kulturellen und beruflichen Leben ist nur in einem Prozess erreichbar, der (1) längere Zeit dauert, (2) eine Mehrzahl von beteiligten Personen und verschiedene Dienste, formelle und informelle Hilfen einbezieht und (3) nebeneinander wie nacheinander gesundheitliche, soziale und beschäftigungs- oder berufsbezogene Aspekte aufweist. Soll dieser Prozess, der insgesamt Rehabilitation ausmacht, zielwirksam erfolgen, wird ein Steuerungsverfahren für den Einzelfall gebraucht, das die Gestaltung der Abläufe, der Koordination und der Zusammenarbeit zum Inhalt hat.«

Wolf Rainer Wendt, Prozessoptimierung durch Case Management

Internet http://www.inreha.net/downloads/pdf/case_management_wendt.pdf 